\title{
LETTER
}

\section{Letter to the Editor Regarding: A Comprehensive Review on Copemyl ${ }^{\circledR}$}

\author{
Giancarlo Comi · Ferdinando Nicoletti · Pier Luigi Canonico • \\ Diego Centonze
}

Received: September 5, 2018 / Published online: November 10, 2018

(C) The Author(s) 2018

Keywords: Equivalence; Follow-on glatiramer acetate; Glatiramer acetate; Multiple sclerosis

We read the review by Annovazzi et al. [1] with great interest. Glatiramer acetate (GA) after the approval of various regulatory agencies has been reliably used for over two decades as first-line treatment for relapsing forms of multiple sclerosis (MS) based on consistent results of multiple clinical trials [2-5] establishing broad

Enhanced digital features To view enhanced digital features for this article go to https://doi.org/10.6084/ m9.figshare.7212653.

\section{G. Comi ( $\square)$}

Department of Neurology, IRCCS San Raffaele Scientific Institute, Milan, Italy

e-mail: comi.giancarlo@hsr.it

\section{F. Nicoletti}

Department of Physiology and Pharmacology, University of Rome "La Sapienza", Rome, Italy

F. Nicoletti

IRCCS Neuromed, Pozzilli, IS, Italy

P. L. Canonico

DISCAFF Department, University of Piemonte

Orientale "Amedeo Avogadro", Novara, Italy

D. Centonze

Neurology and Neurorehabilitation Units, IRCCS

Neuromed, Pozzilli, IS, Italy

D. Centonze

Department of Systems Medicine, University of Rome Tor Vergata, Rome, Italy consensus among general neurologists and MS experts. Assessment of the published preclinical and clinical data of Copemyl ${ }^{\circledR}$ (Mylan N.V., Hatfield, UK), a new member of the follow-on glatiramer acetate (FoGA) class, which include some aspects of its biological and immunological properties, as well as results from the randomized controlled trial that resulted in its authorization, is thus of scientific and medical interest. In their recent review article, Annovazzi et al. reported the published information and marketing authorization decision on Copemyl $^{\circledR}$ [1]. The authors raised questions about some drawbacks and limitations of the GATE (Glatiramer Acetate Clinical Trial to Assess Equivalence with Copaxone) study and its open-label extension [6, 7], the most relevant one being the discrepancy between clinical and magnetic resonance (MRI) end points during the double-blind phase of the study, which invalidates the fundamental study design hypothesis and does not comply with EMA (European Medicines Agency) guidelines [8]. However, several additional gaps and inaccuracies were not included in Annovazzi's review and should be presented to physicians and the scientific community for further resolution in the interest of public health. These are particularly relevant for country-specific decisions on the substitutability of Copaxone ${ }^{\circledR}$ (Teva Pharmaceutical Industries, Petach Tiqva, Israel) with a FoGA, given the concerns for immunogenic 
risk to individual patients. The EMA stated that GA is neither a small molecule nor a biologic drug but is rather a non-biologic complex drug (NBCD) comprised of up to $10^{29}$ polypeptides, sub-sequences of which being antigens that modulate the immune system driving $\mathrm{T}$ cell differentiation towards immune tolerant $\mathrm{T}$ regulatory $\left(\mathrm{T}_{\text {reg }}\right)$ cells at the expense of autoreactive $\mathrm{T}$ helper (Th) 1 and Th17 cells.

Comprehensive characterization of the differences observed in the physicochemical, quality, and biological attributes between Copaxone $^{\circledR}$ and several FoGAs were reported in multiple peer-reviewed publications [9-14], indicating potential risks for altered efficacy, safety, and immunogenicity, but were not addressed in Annovazzi's review. Moreover, some pitfalls and deficiencies in the conduct and outcomes of the single, comparative study performed to demonstrate therapeutic equivalence and comparable immunogenicity of Copemyl $^{\circledR}$ and Copaxone ${ }^{\circledR}[6,7]$ cast doubts about the reported conclusions and implications for everyday practice and the medical equivalence between products.

The above outlined discrepancies in outcomes of the GATE study and deficiencies of the open-label extension further highlight the need to qualify the not-yet fully characterized critical quality attributes of GA and their implications for safety, efficacy, and immunogenicity. Remarkably, it has been demonstrated in several peer-reviewed publications that low-resolution analytical methods, which have been used by the various FoGA manufacturers, failed to reveal differences between FoGAs and Copaxone ${ }^{\circledR}$; however, the use of higher resolution, polypeptide-appropriate, state-of-the-art methodologies showed marked structural differences between FoGAs and Copaxone ${ }^{\circledR}$ $[12,14,15]$. These differences indicate that the amino acid antigenic sequences, length, and amount of peptides are not the same between the two products. When tested vs. Copaxone ${ }^{\circledR}$, Copemyl ${ }^{\circledR}$ has demonstrated differences in surface charge distribution as well as biological activity in potency and cytotoxicity assays $[16,17]$. This raised concerns on the methodology used by FoGAs manufacturers for the analysis of the structural similarities with GA, and also it raised the concrete possibility that FoGAs and GA display different immunogenic profiles. Overall, these findings indicate that the non-similarity in physicochemical attributes of FoGAs compared to Copaxone ${ }^{\circledR}$ is associated with altered biological activity of the antigens and thus may modify the clinical profile, particularly upon chronic exposure and repeated substitutions between the two products.

As a result of their polypeptidic structure, GA and FoGAs may give rise to the formation of anti-drug antibodies, as opposed to small molecules. The active pharmaceutical ingredients in GA and FoGAs are not identical and, similarly to biosimilars with respect to the reference products, their biological and therapeutic equivalence can only be inferred via appropriate bioequivalence and clinical studies. Notably, a similar pharmacokinetic (PK) profile of the products is essential for the approval of biosimilars. However, in the case of GA and FoGAs, for which no PK profile is available, the essential assumption behind the rationale of taking a position in favor of the approval of biosimilars by regulatory authorities of several European countries, that is the ability to demonstrate comparable bioequivalence to the reference product, as well as similar efficacy and safety, cannot be met.

A second point to consider is the comparison of clinical end points across clinical trials. Annovazzi et al. reviewed the randomized, multicenter, double-blind, active, and placebocontrolled phase 3 GATE trial $[6,7]$. The structure and sample size in the 9-month core study and its 15-month open-label extension [7], based upon the European/Canadian MRI study with Copaxone ${ }^{\circledR}$ [3] conducted two decades ago, were originally planned to demonstrate that the effect of the incorrectly defined "generic glatiramer acetate (GTR)" on the MRI disease activity is equivalent to that of Copaxone ${ }^{\circledR}$, and that the safety profile and antibody formation during the core study and its open-label extension do not significantly differ between the two products. This, according to Annovazzi et al., would support the overall claim for therapeutic equivalence suggesting that the efficacy and safety will be retained following a switch from Copaxone ${ }^{\circledR}$ to GTR [1]. 
Despite numerically similar estimates observed between GTR and Copaxone ${ }^{\circledR}$ in the primary end point (total number of gadoliniumenhancing lesions during months 7, 8, and 9 was $9.5 \%$ higher in the GTR group compared with Copaxone ${ }^{\circledR}$ ), and that this was within the pre-specified equivalence margins, we believe that the design and the specific outcomes of the GATE study do not fully substantiate the claim for therapeutic equivalence. The issue of the margins requires special attention. For the GATE study, the upper limit of the margin was set at $50 \%$ of the value of the ratio in mean number of gadolinium-enhancing (GdE) lesions between placebo and Copaxone ${ }^{\circledR}$ observed in the European/Canadian trial in months 7-9. The value of $50 \%$ was chosen because it is possible to estimate the expected effect on relapses from an observed effect on MRI lesions, according to Sormani's equation [18]. Moreover, the GATE study showed no effect in absolute risk reduction (ARR) for Copaxone ${ }^{\circledR}$ versus placebo despite "equivalence" with GTR in treatment effect in reducing GdE lesions, thus invalidating the use of Sormani's equation for the association between the treatment effect achieved on MRI and the treatment effect achieved on relapses, upon which the comparability exercise of therapeutic equivalence (effect on MRI effect on relapses) of the GATE study was based. The authors of the review correctly state that the current EMA guidelines on multiple sclerosis [8] accept brain MRI active lesions as a primary end point for clinical trials aiming to demonstrate the similarity of two products in the context of biosimilar and generic applications. However, it is also stated that "In addition, clinical outcomes such as relapse rate or percentage of relapse-free patients should be used as secondary end points in support of the MRI outcomes. These principles are also valid in the context of a generic application". In the GATE study, Sormani's equation [18] was clearly violated: no relationship has been demonstrated between the treatment effect on MRI and relapses for Copaxone ${ }^{\circledR}$. The authors explain this failure by the small sample of the placebo arm. However, it is quite curious that the value of relapse rate was almost the same in the glatiramer arm (0.40) and in the placebo arm (0.38), while in the European/ Canadian study the values in the two arms were 0.52 and 0.73 , respectively, a significant $29 \%$ reduction of relapse rate in the GA arm. The relapse rate was clearly higher in the European/ Canadian trial; however, the sample size was the opposite: 441 patients in the GATE study versus 239 patients in the European/Canadian trial, not considering the Copemyl ${ }^{\circledR}$ arm. To explain the absence of any effects on relapses the authors claim that also in the PRECISE study (Evaluate Early Glatiramer Acetate Treatment in Delaying Conversion to Clinically Definite Multiple Sclerosis of Subjects Presenting with Clinically Isolated Syndrome) [19] performed in the clinically isolated syndromes, "the effect of GA on clinical activity emerges over placebo after at least 6 months of treatment"; that is a completely wrong interpretation of the results: the two curves of freedom from a second attack separate after the first month and the effect size of GA versus placebo is so large that already at month 6 the difference is statistically significant, which means that a 6-month study duration was sufficient to demonstrate the effect of the drug on relapses.

Moreover, it is hard to understand why the mean number of enhancing lesions in the original placebo arm decreases from 2.5 at baseline to about 0.8 in the third trimester, then it increases to about 1.7 at month 12 after 3 months of treatment with Copemyl ${ }^{\circledR}$ to finally drop again to 0.9 at month 24 . This means that the mean number of enhancing lesions does not change from the end of the double-blind phase to the end of the extension phase when patients shift from the placebo to the Copemyl ${ }^{\circledR}$ treatment. The extension phase failed to confirm the effect of Copemyl ${ }^{\circledR}$ on the primary end point.

A third point concerns the pitfalls of an open-label study design to adequately address any novel comparable efficacy or safety claims. In order to demonstrate long-term "medical equivalence" of GA and GTR, comparability between treated groups must be formally tested [7]. However, in the open label phase of GATE no statistical comparisons (sample size calculation, selection of adequate end points, determination of the magnitude of the differences 
which allow to accept or reject "medical equivalence") were originally planned prior to initiating the GATE study to allow an adequate assessment of the differences in efficacy between the patients who were switched from Copaxone ${ }^{\circledR}$ to GTR and those patients who were treated only with GTR throughout the entire study. Furthermore, the open nature of the extension phase introduced uncertainty and uncontrolled placebo effect in reporting the rate of emerging safety issues. More importantly, the study did not address the practical clinical situations, such as repeated switching between similar but yet not identical pharmaceutical products (Copaxone $^{\circledR}$ to GTR and GTR to Copaxone ${ }^{\circledR}$ ), and particularly the uncertainty about switching from GTR to Copaxone in patients who had inadequate responses to GTR treatment.

In addition to the foregoing methodological problems, we find some of the conclusions contained in the paper questionable. Annovazzi et al. concluded that the incidence and titer of total immunoglobulin G (IgG) anti-GA antibodies were comparable between the two glatiramoids [1]. Moreover, switching from Copaxone $^{\circledR}$ to Copemyl ${ }^{\circledR}$ did not affect total IgG anti-GA antibodies titers. While the incidence and titers of IgG anti-GA antibodies are of great importance in defining one aspect of immunogenicity, the complex nature of antiGA antibodies also requires evaluating the time pattern of IgG anti-GA antibody isotypes and the emergence of anti-GA IgE antibodies that may affect patients' safety or tolerability of treatment. It is extremely important from a clinical standpoint to establish whether, similarly to Copaxone ${ }^{\circledR}$, Copemyl ${ }^{\circledR}$ does not induce neutralizing anti-drug antibodies. This fundamental question is not addressed in the GATE study and in its open-label phase.

Finally, in the open-label extension [7], a similar mean number of GdE lesions was observed for Copaxone ${ }^{\circledR} /$ Copemyl $^{\circledR}$ patients and Copemyl ${ }^{\circledR} /$ Copemyl ${ }^{\circledR}$ patients $(0.6-0.7$ in both groups), whereas placebo/Copemyl ${ }^{\circledR}$ patients showed a higher mean number of GdE lesions at the 12-month scan (1.7). This finding in the placebo group upon switching to Copemyl ${ }^{\circledR}$ suggests a rebound effect on MRI activity.
This requires explanation, as it raises concerns about the validity of the MRI measures after switching.

\section{ACKNOWLEDGEMENTS}

Funding. No funding or sponsorship was received for the publication of this Letter.

Authorship. All named authors meet the International Committee of Medical Journal Editors (ICMJE) criteria for authorship for this article, take responsibility for the integrity of the work as a whole, and have given their approval for this version to be published.

Disclosures. Prof. Giancarlo Comi has received in the past year personal compensation for consulting services and/or speaking activities from Teva, Novartis, Sanofi Genzyme, Merck Serono, Biogen, Roche, Almirall, Celgene, Forward Pharma, Medday. Prof. Ferdinando Nicoletti has received in the last 3 years consultancy fees from Biogen, Lundbeck, Novartis, Alfa-Sigma, FB-Health, Roche, Otsuka, Janssen, and Teva. Prof. Pier Luigi Canonico has received in the last 3 years honoraria, consultancy fees or paid testimony from AbbVie, Amgen, Amicus Therapeutics, Angelini, Astra Zeneca, Biogen, Biomarin, Boheringer Ingelheim, Celgene, CSL-Behring, Daiichi Sankyo, Eli Lilly, Incyte Biosciences, Intercept, Jazz Pharmaceuticals, Laboratoire CTRS, Leo Pharma, Lundbeck, Menarini, Merck, MSD, Novo Nordisk, Otsuka, Roche, Sage Therapeutics, Sanofi, Shire, Takeda, Tesaro, Teva, UCB. Prof. Diego Centonze is an Advisory Board member of Almirall, Bayer Schering, Biogen, GW Pharmaceuticals, Merck Serono, Novartis, Roche, Sanofi-Genzyme, Teva and received honoraria for speaking or consultation fees from Almirall, Bayer Schering, Biogen, GW Pharmaceuticals, Merck Serono, Novartis, Roche, Sanofi-Genzyme, Teva. He is also the principal investigator in clinical trials for Bayer Schering, Biogen, Merck Serono, Mitsubishi, Novartis, Roche, Sanofi-Genzyme, Teva. His preclinical and clinical research was supported by grants from 
Bayer Schering, Biogen Idec, Celgene, Merck Serono, Novartis, Roche, Sanofi-Genzyme and Teva.

Compliance with Ethical Guidelines. This article is based on previously conducted studies and does not contain any studies with human participants or animals performed by any of the authors.

Open Access. This article is distributed under the terms of the Creative Commons Attribution-NonCommercial 4.0 International License (http://creativecommons.org/licenses/ by-nc/4.0/), which permits any noncommercial use, distribution, and reproduction in any medium, provided you give appropriate credit to the original author(s) and the source, provide a link to the Creative Commons license, and indicate if changes were made.

\section{REFERENCES}

1. Annovazzi P, Bertolotto A, Brescia Morra V, et al. A comprehensive review on Copemyl®. Neurol Ther. 2017;6(2):161-73.

2. Bornstein MB, Miller A, Slagle S, et al. A pilot trial of Cop 1 in exacerbating-remitting multiple sclerosis. N Engl J Med. 1987;317(7):408-14.

3. Comi G, Filippi M, Wolinsky JS. European/Canadian multicenter, double-blind, randomized, placebo-controlled study of the effects of glatiramer acetate on magnetic resonance imaging-measured disease activity and burden in patients with relapsing multiple sclerosis. European/Canadian Glatiramer Acetate Study Group. Ann Neurol. 2001;49(3):290-7.

4. Johnson KP, Brooks BR, Cohen JA, et al. Copolymer 1 reduces relapse rate and improves disability in relapsing-remitting multiple sclerosis: results of a phase III multicenter, double-blind placebo-controlled trial. The Copolymer 1 Multiple Sclerosis Study Group. Neurology. 1995;45(7):1268-76.

5. Khan O, Rieckmann P, Boyko A, Selmaj K, Zivadinov R. Three times weekly glatiramer acetate in relapsing-remitting multiple sclerosis. Ann Neurol. 2013;73(6):705-13.

6. Cohen J, Belova A, Selmaj K, et al. Equivalence of generic glatiramer acetate in multiple sclerosis: a randomized clinical trial. JAMA Neurol. 2015;72(12):1433-41.

7. Selmaj K, Barkhof F, Belova AN, et al. Switching from branded to generic glatiramer acetate: 15-month GATE trial extension results. Mult Scler. 2017;23(14):1909-17.

8. European Medicines Agency. Guideline on clinical investigation of medicinal products for the treatment of multiple sclerosis. EMA/CHMP/771815/ 2011, Rev. 2. 2015. http://www.ema.europa.eu. Accessed Nov 17, 2017.

9. Hasson T, Kolitz S, Towfic F, et al. Functional effects of the antigen glatiramer acetate are complex and tightly associated with its composition. J Neuroimmunol. 2016;290:84-95.

10. Kolitz S, Hasson T, Towfic F, et al. Gene expression studies of a human monocyte cell line identify dissimilarities between differently manufactured glatiramoids. Sci Rep. 2015;5:10191.

11. Kolitz S, Laifenfeld D, Fowler K, et al. Similarities and differences in the gene expression profiles of Glatopa and Copaxone. ECTRIMS 2016. Mult Scler J. 2016;22(Suppl 3):170.

12. Komlosh A, Pinkert D. Method of determining the molecular weight distribution of glatiramer acetate using multi-angle laser light scattering (MALLS). Publication no. WO2016176649 A1. Application no. PCT/US2016/030277. Google Patents; 2016.

13. Laifenfeld D, Hasson T, Kolitz S, et al. Similarities and differences in the gene expression profiles of Copaxone and Polimunol. ECTRIMS 2016. Mult Scler J. 2016;22(Suppl 3):175.

14. Wells-Knecht K, Denny R, Hasson T, et al. Testing the limits of ion mobility mass spectrometry to compare a nonbiological complex drug product and purported generics-a case study with Copaxone. Spectroscopy. 2017;15(2):29-34.

15. Komlosh A, Hasson T, Wells-Knecht K, et al. Similarities and differences in properties of glatiramer acetate (Copaxone ${ }^{\circledR}$, Teva) versus polimunol (Synthon) using standard and emerging technologies. Eur J Neurol. 2016;23(Suppl):199.

16. Komlosh A, Krispin R, Papir G, et al. Surface charge distribution, an attribute linked with immunogenicity of nanoparticles, is different for follow-on glatiramer acetate products approved in EU, Russia, Latin America, and USA compared with Copaxone. ECTRIMS 2017. Mult Scler J. 2017;23(Suppl 3):869.

17. Timan B, Komlosh A, Beriozkin O, et al. Physicochemical and biological characterization of the European follow-on glatiramer acetate product as 
compared to Copaxone. ECTRIMS 2017. Mult Scler J. 2017;23(Suppl 3):869.

18. Sormani MP, Bonzano L, Roccatagliata L, Mancardi GL, Uccelli A, Bruzzi P. Surrogate endpoints for EDSS worsening in multiple sclerosis. A meta-analytic approach. Neurology. 2010;75(4):302-9.
19. Comi G, Martinelli V, Rodegher M, et al. Effect of glatiramer acetate on conversion to clinically definite multiple sclerosis in patients with clinically isolated syndrome (PreCISe study): a randomised, double-blind, placebo-controlled trial. Lancet. 2009;374:1503-11. 